\title{
The Relationship between Chaotic Characteristics of Physiological Signals and Emotion Based on Approximate Entropy
}

\author{
Nie Chun-yan \\ Electronic Information Institute \\ Changchun University \\ Changchun, China, 130022 \\ e-mail: ncy66@163.com
}

\author{
Sun Hai-xin \\ Electronic Information Institute \\ Changchun University \\ Changchun, China, 130022 \\ e-mail:haixin_s@hotmail.com
}

\author{
Wang Ju \\ Electronic Information Institute \\ Changchun University \\ Changchun, China, 130022 \\ e-mail: youyou8221@yahoo.com.cn
}

\begin{abstract}
Emotion recognition is an important part in affective computing. It is the basis of building a harmonious man-machine environment. Respiratory (RSP) signal and electrocardiogram (ECG) signal are one of the main study objects in the emotion recognition based on physiological signal. The variations of the RSP signal and the ECG signal is one of the true performances of the human emotions. Through the analyses of the RSP signal and the ECG signal, we can recognize the inner emotion variations of human beings. This lays the foundation for the system modeling of emotion recognition. In this paper, we study the approximate entropy extraction of the physiological signals and analyze the chaotic characteristics and frequency domain characteristics of the approximate entropy under different emotions. The study results show that the different emotion status is corresponding to different approximate entropy and different variations in the frequency domain.
\end{abstract}

Keywords-Respiratary signal; Electrocardiography signal; Emotion Recognition; Approximate entropy

\section{INTRODUCTION}

Emotion recognition is one of the most important parts in the field of affective computing. The research contents of the emotion recognition involve affective speech, facial expression, gesture expression, emotion recognition of the physiological signal etc. [1]. Among the contents mentioned above, emotion recognition of the physiological signal is the most difficult. The studies on emotion recognition of facial expression and speech are the complex patterns expressed by the behavior and the body, whether willing or unwilling. Besides, there are large amounts of researchers engaged in this field and the technologies are relatively mature [2]-[5]. However, these methods cannot detect the potential emotion. The physiological signal is a kind of bioelectric signal which is generated by the internal organs of the human being accompanying the variations of the emotion. It can reflect the real-time variations of the emotion more objectively and truly and further improve the correct rate of the emotion recognition. The emotion recognition based on physiological signal can be applied in the fields of psychological rehabilitation therapy and polygraph and has a broad application prospects.

The organization of the paper is as follows: In Section 2, we describe the relationship between the physiological signal and emotion. In Section 3, we give the acquisition process of the physiological signal. In Section 4, we give the definition of the approximate entropy and analyze the solution of the approximate entropy. In Section 5, we simulate on the RSP signal and the ECG signal and analyze the approximate entropy and the characteristics in the frequency domain respectively.

\section{RELATIONSHIPS BETWEEN PHYSIOLOGICAL SIGNAL AND EMOTION}

The traditional physiological signals used in the emotion recognition are respiratory (RSP) signal, electrocardiogram (ECG) signal, electroencephalogram (EEG) signal, skin response (SE), electromyography (EMG), blood volume pulse (BVP), skin temperature (SKT) and etc. Since the physiological variations are dominated by the endocrine system and the autonomic nervous system, it isn't dominated by the subjective conscious control. Through the physiological signal analysis method, we can obtain more objective and real data. If we can find some characteristic or the combination of the characteristics which represent the specific emotion, we can use these characteristics for the emotion recognition and the harmonious human-machine affective interaction is achieved. Considering the implementation of collecting physiological signal and the complexity and effectiveness of the experiments, we recognize the emotion through two kinds of physiological signals: RSP signal and ECG signal.

Respiration is the process of interchanging air between the body of human being and external environment. It is usually affected by the workload of the body. With the increase of the workload, the energy metabolism and oxygen needed by the body are increased. Furthermore, respiration can describe the activities of the autonomic nervous system under emotional reaction and mental workload conditions. With the variations of the emotional state, the respiration activities may change in the aspects of speed and depth. Intense pain reaction may leads to fast and deep breath. A sudden panic may result in a temporary interruption of the breath. In the state of exultation or grief, the respiratory spasm phenomenon will occur. Therefore, the RSP signal plays an important role in recognizing the emotional states. For example, deep and fast breath indicates an excited state and the corresponding emotion may be happy or angry but deep and slow breath indicates a relax state. Shallow and short breath indicates a nervous state but shallow and slow breath indicates a quiet or negative state [5]. 
Furthermore, physiological signal is an unstable signal generated by the complex life entity. It is different from the ordinary signal in the aspects of signal characteristics, detection methods and processing technologies. Since it is affected by many factors of the human being, the physiological signal has unique characteristics of the weak signal, strong noise and low frequency range. Except that the spectrum of the ECG signal is high, the spectral of other physiological signals are low. Besides, the ECG has the characteristics of randomness and nonstationarity.

\section{PhysiologicAl SignAl ACQUISITION}

When the physiological signal is employed in emotion recognition, it is vital to acquire emotional physiological signals since it has a direct impact on the subsequent effect of emotion recognition. All the research works are on the basis of emotional physiological signals. Hence, effective schemes need to be taken to acquire emotional physiological signals. Normally, all the testees who are healthy, no heart disease and a history of mental illness are required to join the tests voluntarily.

The acquisition process of the RSP signal is as follows. Before the test, we let the testees have a rest to achieve a calm state as far as possible and let them feel relax and not curious to the test. Then the test starts. First, the collection device is connected and the breathing belts for acquiring RSP signals are entwined around the thorax of the testee. Next, we observe the recorded RSP signals in the computer to see whether the signal is normal. After that, we play movie clips for testing. The testees are needed to watch four movie clips with different emotions. After each clip, the testees whose emotions are excited by the movie clips need to fill the emotional questionnaire truly. The questionnaire involves two aspects: the category and the intensity of the excited emotions. After the test, we analyze whether the testees show the true emotions during the movie clips by comparing the questionnaire and the observed signal. When selecting the RSP signal data which excite emotion effectively, we take the observation of the expression behavior and the subjective evaluation results of the testees as the references and observe whether the emotions of the testees showed in the questionnaire are in accordance with the emotions need to be expressed truly. The selected data have the excited emotion intensity of middle level or above the middle level. Finally, one hundred groups of RSP signal data which excite emotion effectively are selected as the original database for the RSP signal, including surprise data, angry data, sorrow data and happy data, each of which are twenty five groups.

The acquisition process of the ECG signal is as follows. The instruments used in the test are the multi-channel physiological recorder MP150 of Biopac Corporation. Before the test, the electrode sensors are connected to the body of the testees in a non-intrusive manner. Among the sensors, the two electrodes are connected to the twists of the testee and the other electrode is connected to the right ankle of the testee for grounding. After all the preparatory works, the testee stays in a closed and quiet room alone. The tester uses two computers outside the room. One computer is used for playing movies and the other one is used for acquiring the emotional ECG signals. The movies with different emotions are played in a certain order. After each movie, the testee should fill the emotional experience questionnaire according to the tips on the screen to indicate which kind of emotion is excited by the movies and the degree of the excitation. At the end of the tests, the effective excited emotional data are selected from the tested data according to the questionnaire and the noise interference level during the collection of the physiological signal.

\section{CHAOTIC CHARACTERISTIC PARAMETER- APPROXIMATE ENTROPY}

Nonlinear dynamics is an emerging interdiscipline whose developments open a broad prospect for the studies in various fields. In general, the body of the human being is in a stable and chaotic state, which is an equilibrium state in reality. However, different diseases often result in the imbalances of the chaotic state. In the nonlinear dynamics, the chaotic characteristic parameters can be used to measure the complexity of the physiological signals. The smaller the complexity is, the lower the chaos degrees are. The combination of chaos theory and biomedical engineering promotes the in-depth study in biomedical problems and provide a new way for studying physiological signal, whose parameter variations are difficult to describe by classical figures accurately.

Approximate entropy, which is one of the chaotic characteristic parameters, represents the complex degree of a data sequence and its value is proportional to the complex degree. Usually, the value of approximate entropy is a nonnegative number. More complex the sequence is, larger the value of the approximate entropy is. Approximate entropy has a strong ability to characterize the complexity of the signals, such as the chaotic signals and the random signals. The amount of the data required to calculate the approximate entropy is small, so it is suitable for analyzing short time data. Besides, approximate entropy has a strong anti-noise capability.

Let the length of the data sequence $\{x(i)\}$ equal to $N$, the steps of solving approximate entropy are as follows[6]-[7].

(1) Arrange the sequence $\{x(i)\}$ into a vector of length $m$ in a certain order:

$$
X(i)=\{x(i), x(i+1), \cdots, x(i+m-1)\}
$$

(2) Let the distance between $\{X(i)\}$ and $\{X(j)\}$ is

$$
d(X(i), X(j))=\max _{i=1,2, \cdots, m}|x(i+k-1)-x(j+k-1)|
$$

(3) Given a threshold $r$, count the amount of $d(X(i), X(j))$ smaller than $r$ and calculate the ratio between it and the total number of the distance for any $i$ :

$$
C_{i}^{m}(r)=\frac{1}{N-m+1}\{N u m(d(X(i), X(j))<r\}
$$

(4) After taking the logarithm of $C_{i}^{m}(r)$, calculate the average value for all $i$ :

$$
\theta^{m}(r)=\frac{1}{N-m+1} \sum_{i=1}^{N-m+1} \ln C_{i}^{m}(r)
$$


(5) Calculate $\theta^{m+1}(r)$;

(6) Calculate the approximate entropy:

$$
\theta^{m}(r)-\theta^{m+1}(r)
$$

\section{SimULATIONS AND ANALYSES}

\section{A. Approximate Entropy and Frequency Domain Analysis for RSP signal}

The variation of the RSP signal is one of the true emotion performances of human being. Through acquiring ideal RSP signal data which are in accordance with the real environment, we can extract the effective emotional physiological characteristics, analyze the RSP signal and recognize the inner emotional variations. In this paper, we calculate the approximate entropy values of the RSP signal and select one hundred groups RSP signals of exciting emotion effectively, including surprise data, angry data, sorrow data and happy data, each of which are twenty five groups. The approximate entropy values of the RSP signal under different emotions are given in Fig.1.

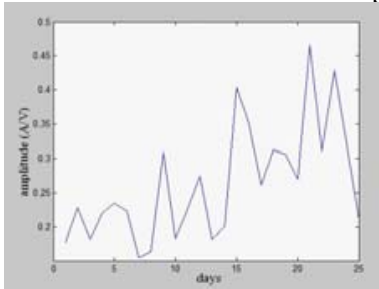

(a) joy

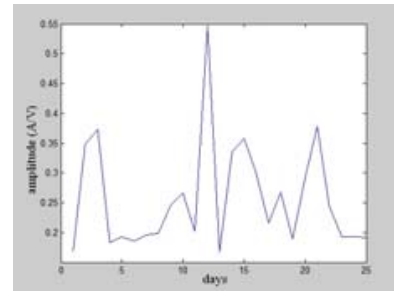

(c) sadness

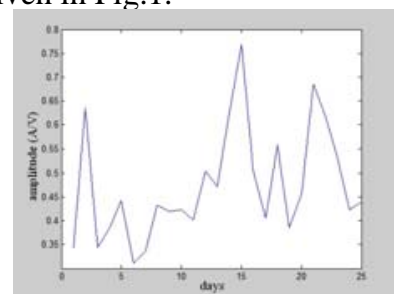

(b) anger

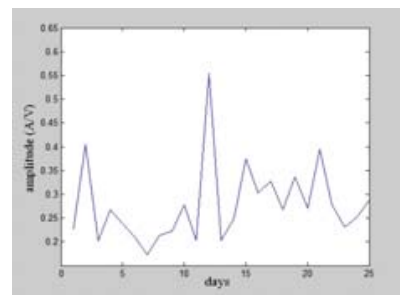

(d) pleasure
Figure 1. The approximate entropy of the RSP signal under different emotions.

As illustrated in Fig.1, after removing some large errors, the ranges of the approximate entropy value of the RSP signal are different. The approximate entropy ranges for joy, anger, sadness and pleasure emotions are from $0.15 \sim 0.45$, $0.28 \sim 0.78,0.15 \sim 0.55,0.15 \sim 0.55$, respectively. It is obvious that the approximate entropy value of the anger emotion is larger than that of other emotions. Hence, we can identify the anger emotions through the value of the approximate entropy.

Next, we analyze the RSP signal data of twenty five days in the frequency domain. The simulation results are given in Fig. 2. From the figures, we find that the breathing frequency range of the anger emotion is from $100 \sim 200 \mathrm{~Hz}$, but the breathing frequencies of other emotions are from $50 \sim 100 \mathrm{~Hz}$. So the breathing frequency of the anger emotion is higher than that of other emotions. Besides, the amplitudes of the RSP signal under different emotions are in the same level.
Hence, we can identify the emotional states according to the frequency ranges of the RSP signal.

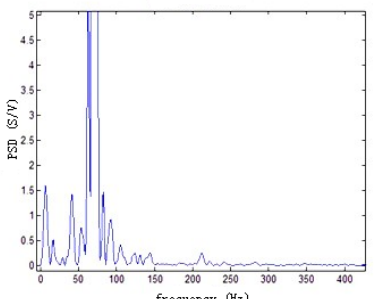

(a) joy

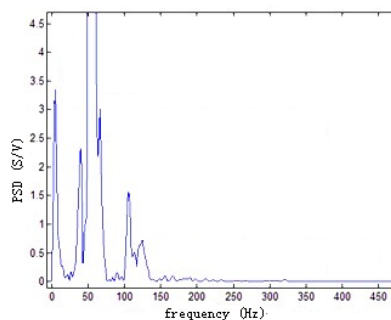

(c) sadness

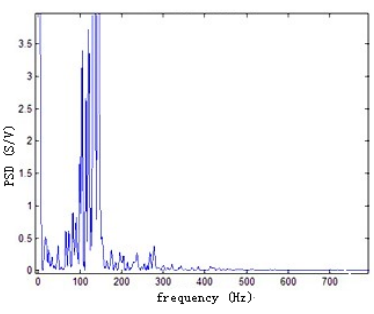

(b) anger

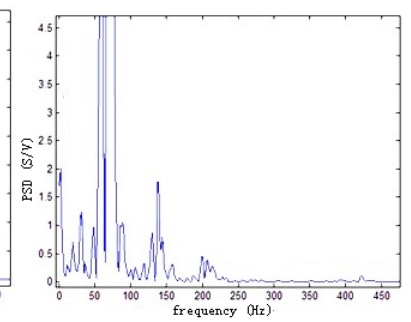

(d) pleasure

Figure 2. The frequency domain waveforms of the RSP signal under different emotions.

\section{B. Approximate Entropy and Frequency Domain Analysis for ECG signal}

ECG signal is such a signal that is generated by the myocardial cells during the human heart beating. In this paper, we collect one hundred groups emotional ECG signals of exciting emotion effectively, including surprise data, angry data, sorrow data and happy data, each of which are twenty five groups. After the analyses, we obtain the following data. For the ECG signals, the approximate entropy ranges for joy, anger, sadness and pleasure emotions are from 0.15 0.65, 0.14 0.55, 0.13 0.62, 0.12 0.48, respectively. The data above show that the approximate entropy values of the ECG signal under different emotions are approximate. Hence, we need other methods combing with the approximate entropy of the ECG signal to identify the emotional state. Due to the limited space, we don't give the approximate entropy curves of the ECG signals in this paper.

Furthermore, we analyze the ECG signal data of twenty five days in the frequency domain. The simulation results are shown in Fig. 3. From the figures, the frequency ranges of ECG signal for joy, anger, sadness and pleasure emotions are almost the same. However, the average amplitudes of ECG signal under joy and pleasure emotions are higher than those under anger and sadness emotions. Meanwhile, the average amplitudes of ECG signal under anger and sadness emotions are much higher than those under joy and pleasure emotions in the frequency range of $0 \sim 200 \mathrm{~Hz}$, which shows that the ECG signals under anger and sadness emotions have a larger variation range. 


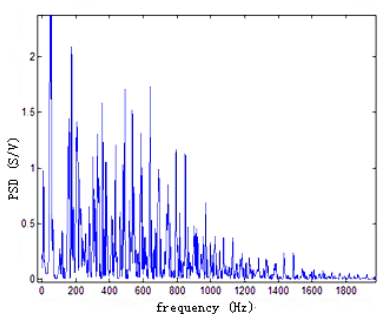

(a) joy

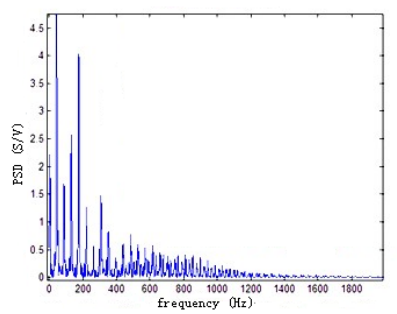

(c) sadness

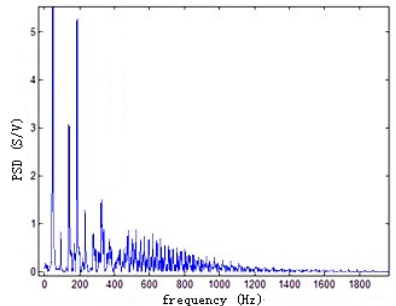

(b) anger

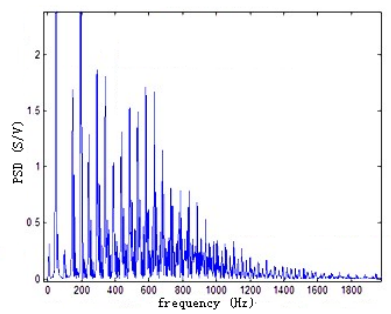

(d) pleasure
Figure 3. The frequency domain waveforms of the ECG signal under different emotions.

\section{CONCLUSIONS}

Physiological signals can reflect the real changes of the emotional states of the human being. In this paper, we study the variations of the RSP signal and the ECG signal under different emotions. From the theoretical analysis and the simulation results, we have the following conclusions. First, for the RSP signal, the approximate entropy value under anger emotions is higher than that under other three emotions. Hence, we can recognize the anger emotions through the approximate entropy value. In the frequency domain, we can recognize the emotional states according to the different frequency ranges of the RSP signal. Second, for the ECG signal, according to the nonlinear analysis, the approximate entropy values of ECG signal under the four different emotions are almost the same. Therefore, we need other methods combing with the approximate entropy of the ECG signal to identify the emotional states. From the frequency domain analysis, the variations of the anger and sadness emotions are larger than those of the joy and pleasure emotions.

\section{ACKNOWLEDGMENT}

This work is partially supported by Jilin Province Science Foundation Grant No. 201215110 and " $12^{\text {th }}$ FiveYear Plan" subject of the Jilin Provincial Department of Education Science and Technology Research Grant No.2012242.

\section{REFERENCES}

[1] Picard R W.Affective computing[M]. Cambridge, MA: The MIT. Press, 1997.

[2] Picard R W, Vyzas E, Healey J. Toward machine emotional intelligence: Analysis of affective physiological state[J]. IEEE Transactions Pattern Analysis and Machine Intelligence, 2001, 23(10): 1175-1191.

[3] Nie Chunyan, Li Rui, Wang Ju. Application of Chaotic Characteristics about Multiple Physiological Signals in Emotion Recognition. 2012 Second International Conference on Electric Information and Control Engineering. China, 2012, 2688-2691.

[4] Jing Zhai and Armando Barreto. Stress Deteetion in ComPuter Users Based on Digital Signal Processing of Noninvasive Physiologieal Variables. Proeeedings of the 28th IEEE EMBS Annual Intemational Conferenee.NewYork, USA.2006, 1355-1358.

[5] Key research areas of the Japanese Ministry of Education: Emotional Information Processing, Information Science, Psychology Research. The outcome report, 1999.

[6] LV Jin-hu. Chaotic time series analysis and its application[M]. Wu Han: Wuhan University Press. 2001.

[7] Zou Zhuoying, Xu Xuetian, Chen Yunqia. Analysis of Physiological Signals Based on Nonlinear Methods[J]. Mikro-computer Information, 2009, 25(3): 163-165. 